\title{
GEOMETRICAL REALIZATION OF ISOMORPHISMS BETWEEN PLANE GROUPS
}

\author{
BY A. M. MACBEATH
}

\author{
Communicated by L. Bers, February 5, 1965
}

Let $G$ denote the group of all isometries of the hyperbolic plane. If we take the Poincaré model, the subset of the complex plane with $g z>0$, and the metric $|d z| / g z$, then $G$ consists of orientation-preserving maps

$$
w=\frac{a z+b}{c z+d}, \quad a, b, c, d \text { real } a d-b c=1
$$

and orientation-reversing maps

$$
w=\frac{a \bar{z}+b}{c \bar{z}+d}, \quad a, b, c, d \text { real } a d-b c=-1 .
$$

We denote the upper half-plane by $D$, so that $[G, D]$ is a transformation group with the topology on $G$ and $D$ induced by the parameters $a, b, c, d, z$.

If $H$ is a discrete subgroup of $G$, we call the transformation group $[H, D]$ a non-euclidean crystallographic group (NEC group). The structure of those NEC groups which consist entirely of orientationpreserving maps (Fuchsian groups) has been well-known for some time, but the structure of general NEC groups has only recently been obtained [3]. The aim of this note is to prove the following

TheOREM. Let $[H, D]$ be an NEC group with compact orbit-space $D / H$. Let $\alpha: H \rightarrow H$ be an automorphism of the group $H$. Then the automorphism $\alpha$ can be geometrically realized, i.e., there is a homeomorphism $t: D \rightarrow D$ such that, for all $h \in H$, if $h^{\alpha}$ denotes the image of $h$ under the automorphism $\alpha$, we have

$$
t(h z)=h^{\alpha} t(z) .
$$

The NEC groups include, as special cases, the fundamental groups of all compact surfaces except the sphere, projective plane, torus and Klein bottle. For such groups, the result is due to Nielsen, and can be re-expressed in the form:

Every isomorphism between the fundamental groups of two compact surfaces can be induced by a homeomorphism of one surface on the other.

For Fuchsian groups, the result has recently been proved by Zieschang [4], using combinatorial methods. 
In the present proof, we shall assume the truth of Nielsen's theorem, and deduce the more general result by using an idea of Ahlfors [1] from the theory of quasiconformal mappings.

If $H$ is not a Fuchsian group, then it contains a Fuchsian group as a subgroup of index 2, the subgroup of orientation-preserving transformations. By a theorem of Fox [2] the Fuchsian group contains, as a subgroup of finite index, a group which is a surface group, the fundamental group of a surface. Since every subgroup of finite index contains a normal subgroup of finite index, there is a subgroup $N$ of $H$ which is normal in $H$ and which is the fundamental group of an orientable surface.

By Nielsen's theorem, applied to the surfaces $D / N, D / N^{\alpha}$, there is a homeomorphism $t: D \rightarrow D$, such that, for all $n \in N$,

$$
t(n z)=n^{\alpha} t(z) \text {. }
$$

Among all such homeomorphisms there is a unique extremal quasiconformal homeomorphism. Assume, then, that $t$ is this unique mapping. Let $h \in H$. Define a new mapping $t^{\prime}: D \rightarrow D$ by the equations

$$
t^{\prime}(z)=h^{\alpha} t\left(h^{-1} z\right) \text {. }
$$

Since $N$ is a normal subgroup we have from (1)

$$
t^{\prime}(n z)=h^{\alpha} t\left(h^{-1} n h \cdot h^{-1} z\right)=h^{\alpha}\left(h^{-1} n h\right)^{\alpha} t\left(h^{-1} z\right)=n^{\alpha} t^{\prime}(z) .
$$

Thus $t^{\prime}$ also satisfies equation (1). Now $t^{\prime}$ is obtained from $t$ by preceding and following it by conformal mappings. Thus the maximal dilatation of $t^{\prime}$ is equal to the maximal dilatation of $t$, so $t^{\prime}$ is also an extremal quasiconformal mapping. By the uniqueness theorem, $t^{\prime}=t$, and our theorem follows.

\section{REFERENCES}

1. L. V. Ahlfors, On quasiconformal mappings, J. Analyse Math. 3 (1953/54), 1-58.

2. R. H. Fox, On Fenchel's conjecture about F-groups, Mat. Tidssk. 1952 (1952), 61-65. 1963.

3. H. C. Wilkie, Non-euclidean crystallographic groups, Ph.D. Thesis, St. Andrews,

4. H. Zieschang, Über Automorphismen ebener Gruppen, Dokl. Akad. Nauk SSSR 155 (1964), 57-60.

UNIVERSITY OF BIRMINGHAM, BIRMINGHAM, ENGLAND 